\title{
Investigation of a cleaning procedure for silicon spheres used in the realization and dissemination of the redefined kilogram via combined spectroscopic and gravimetric measurements
}

\author{
Edyta Beyer ${ }^{1, *}$, Michael Borys ${ }^{2}$, Michael Mecke $^{2}$, and Michael Kolbe ${ }^{1}$ \\ ${ }^{1}$ Physikalisch-Technische Bundesanstalt, Abbestr. 2-12, 10587 Berlin, Germany \\ ${ }^{2}$ Physikalisch-Technische Bundesanstalt, Bundesallee 100, 38116 Braunschweig, Germany
}

Received: 4 November 2020 / Accepted: 19 November 2020

\begin{abstract}
The new definition of the SI kilogram requires new methods of realizing this unit. The X-ray crystal density method is a primary realization method and uses silicon spheres. The spheres get cleaned before each measurement, in order to remove surface contaminations and thus reduce their uncertainty contribution to the realization. Therefore, cleaning is an inherent part of the realization and dissemination of the kilogram. A cleaning method for silicon spheres is investigated, concerning its suitability as a part of the realization of the redefined kilogram. Six silicon spheres were used to determine the repeatability of the established cleaning method. Measurements of the spheres' mass and the quantification of their surface layer mass after cleaning were carried out in several cycles resulting in 29 mass and surface measurements. The repeatability of the cleaning method applied shows a standard deviation in the order of two micrograms for both the mass and the surface layer. The cleaning method therefore sufficiently fulfils these requirements.
\end{abstract}

Keywords: Redefined kilogram / silicon spheres / cleaning method / repeatability / mass stability / surface characterization

\section{Introduction}

Since the revision of the SI in May 2019, the kilogram has no longer been defined as the mass of the international prototype of the kilogram. The new definition is now based on natural constants whose numerical values have been fixed with a defined uncertainty of zero. These constants are the Planck constant, the unperturbed ground-state hyperfine transition frequency of ${ }^{133} \mathrm{Cs}$ and the speed of light in vacuum [1-3]. Primary methods are needed for the practical realization of the kilogram definition [2] whereby the X-ray crystal density (XRCD) method is one method of realizing this unit and uses monocrystalline isotopically enriched ${ }^{28} \mathrm{Si}$ spheres. The ${ }^{28} \mathrm{Si}$ spheres then work as primary mass standards. In addition, spheres made of natural silicon can be used as secondary mass standards to disseminate and maintain the kilogram.

This work investigates the short term mass stability of silicon spheres including the influence of handling, as the suitability of silicon spheres as mass standards increases with better stability. The surface of silicon spheres has a crucial influence on their mass stability, which in turn

\footnotetext{
* Corresponding author: edyta. beyer@ptb.de
}

affects their uncertainty budget and appropriate realization intervals. The surface layer (SL) consists of several sublayers. The silicon oxide layer $(\mathrm{OL})$ is itself divided into silicon dioxide and sub oxides $\left(\mathrm{Si}_{2} \mathrm{O}, \mathrm{SiO}, \mathrm{Si}_{2} \mathrm{O}_{3}\right)$ at the interface of the dioxide and the bulk. In addition, there are a carbonaceous contamination layer (CL), and a water layer (WL) [4]. From the structure of the surface layer, it appears that the mass stability of a sphere can be influenced not only by oxide layer growth, but also by the sorption of carbonaceous contaminations and water. Since the spheres are cleaned before each measurement, the repeatability of the cleaning method has been investigated [5-9]. In addition and complementary to the investigations described in [9] the study shall combine the surface analysis with X-ray techniques and gravimetric measurements in order to compare results between these independent methods, document correlations between both methods, identify remaining contaminations on the silicon spheres and support uncertainty analysis for the mass of the surface layer. This study is designed to prove whether the applied cleaning method fulfills the requirements of a realization of the kilogram with smallest uncertainties or requires further improvement. Combined gravimetric measurements and a spectroscopic quantification of the surface layer mass were conducted. A custom instrument for silicon spheres that 

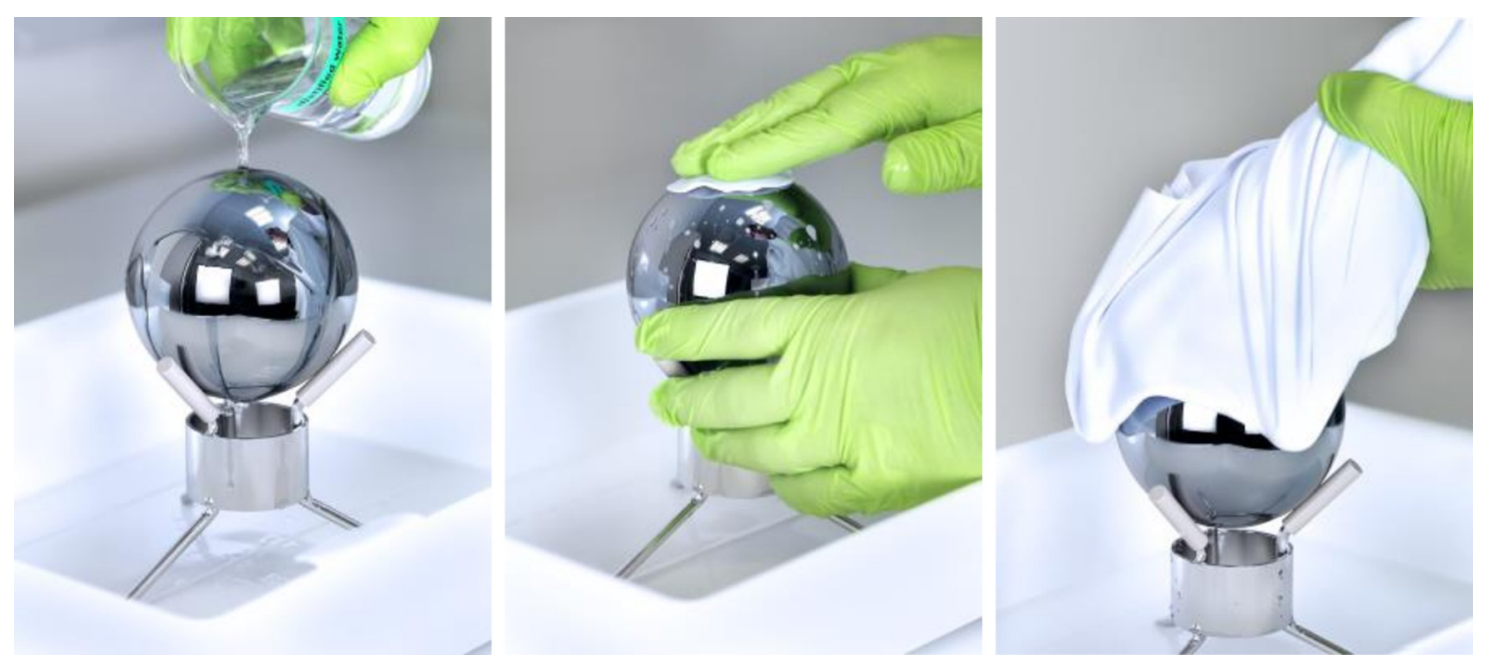

Fig. 1. Selected steps of the cleaning procedure of silicon spheres. From left to right: rinsing with distilled water, washing with cleaning solution and a microfiber pad and picking and the sphere with a microfiber cloth.

combines X-ray fluorescence analysis (XRF) and X-ray photoelectron spectroscopy (XPS) was used for the characterization of the surface layer [10,11]. A mass comparator with a load lock system compatible with the $\mathrm{XRF} / \mathrm{XPS}$ instrument was used for the gravimetric measurements.

\section{Materials and methods}

\subsection{Cleaning procedure for silicon spheres}

The materials used in the cleaning procedure are: ethanol for analysis with a purity of $96 \%$; distilled water; Deconex OP 163 solution (2\%); one large microfiber cloth and one microfiber washing pad for precision cleaning with a knitwear structure, a mesh density of 900 per $\mathrm{cm}^{2}$ and a thickness of $0.72 \mathrm{~mm}$; and powder-free nitrile gloves. The Deconex OP 163 used for cleaning is a pH-neutral, saltfree cleaning concentrate for precision optical components. Its special surfactants completely wet glass surfaces, thus guaranteeing a thorough cleaning and removal of slightly adherent organic and inorganic residues such as fingerprints and dust. Before the actual cleaning begins, the gloves and the sphere-holding device need to be cleaned with Deconex solution and rinsed with distilled water. The clean gloves should not be used to touch anything besides the sphere and the cleaning cloth.

The first step in the cleaning process is to rinse the sphere with distilled water to wash away dust particles from the surface. Otherwise, particles may scratch the sphere during washing. To ensure that the water covers the entire surface of the sphere, the sphere needs to be lifted and turned $180^{\circ}$. A dampened microfiber pad is then used for washing with the Deconex solution. Washing takes place by applying light pressure and moving the microfiber pad in circles. The cleaning pads are used only once. After the gloves have been rinsed with distilled water to remove the surfactant, the sphere itself is placed under a stream of distilled water. Following this, the gloves are rinsed with ethanol and dried. Next, the silicon sphere is rinsed with ethanol. To finish the cleaning, the silicon sphere is lifted with a microfiber cloth and dried off, during which direct contact between the sphere and the gloves is avoided (see Fig. 1). Finally, the sphere is placed in a transport container and is ready to be measured [8].

\subsection{Study of the cleaning procedure}

Six test spheres with the identifiers Si15_12, SiSC01_a, SiSC01_b, SiSC01_c, SiSC01_d and SiSC $\bar{S} 01$ e are used to investigate the repeatability of the cleaning method. All were manufactured in the same way, while five of the spheres (SiSC01_a to SiSC01_e) originated from one silicon crystal and were manufactured in the same time frame. Thus, similar surface properties are expected.

All spheres are cleaned before the measurements are performed, then loaded into a mass comparator in air. The mass comparator is then evacuated and the gravimetrical measurements are performed in vacuum over a period of three to ten days. Following the mass comparisons, the test spheres are transferred to the XRF/XPS instrument for the characterization of the surface layers in a sealed container under vacuum. After the spectroscopic measurements, the spheres are transferred to air, cleaned and loaded into the vacuum transfer system of the mass comparator, and then transferred under vacuum back to the measurement chamber of the mass comparator. Each sphere always returns to its previous measurement position in the comparator. Thus, every sphere has a designated position in the comparator, which does not change during the course of the study.

Two measurement series are performed: the first in April/May 2017 and the second in September/October 2017. Between the completion of the first series in the end of May and the beginning of the second series in the beginning of September 2017, all test spheres are stored in their transport containers in air. 


\subsubsection{Mass measurements}

In addition to the six test spheres, a silicon sphere with the identifier Sm14 is selected as a mass reference. The mass stability of this sphere is known from previous measurements and is checked during the measurements by means of an additional reference sphere, Si12-06. The mass comparison of the spheres is done by a Sartorius CCL1007 vacuum mass comparator in a pressure range from $5 \times 10^{-5}$ to $5 \times 10^{-6} \mathrm{hPa}$. The nominal load of this mass comparator is $1 \mathrm{~kg}$ and it achieves a standard deviation of less than $0.1 \mu \mathrm{g}$ in vacuum. Besides, it is equipped with a vacuum transfer system which is compatible with the load lock of the combined XRF/XPS instrument for surface layer analysis on silicon spheres (see Sect. 2.2.2). In order to minimize possible contaminations during the transfer between the mass comparator and the XRF/XPS instrument, the spheres are transferred under vacuum conditions in a sealed container. From previous measurements, the pressure during the transfer in the sealed container is estimated to be $<0.1 \mathrm{hPa}$. This pressure range is sufficient to stabilize the surface layers on the sphere during the transfer between both systems. The timespan for the transfer of a sphere was approximately thirty minutes. A typical mass stability within $\pm 1 \mu \mathrm{g}\left( \pm 1 \times 10^{-9}\right.$ relative $)$ was observed.

\subsubsection{Surface measurements}

The surface measurements are carried out using an instrument that allows XRF and XPS measurements to be performed on spheres with a diameter of about $93.7 \mathrm{~mm}$ as well as on flat samples. The excitation source is an $\mathrm{Al}$ $\mathrm{X}$-ray tube with a quartz crystal to monochromatize the $\mathrm{Al}$ $\mathrm{K} \alpha$ fluorescence line. A five-axis ball manipulator allows each point on a sphere to be accessible for measurements. The instrument is equipped with a load lock chamber that features a vacuum transfer system to facilitate the transport of the sphere under vacuum [10]. The quantification method relies on a combination of XRF and XPS analysis. For the XRF measurements a reference-based quantification scheme is applied to obtain the mass deposition for oxygen in the surface layer of the sphere. The approach relies on the correlation between the mass deposition of an element and its fluorescence radiation intensity [12].

\subsubsection{XRF measurement of reference samples}

A set of five samples with different oxide layer thicknesses in the nominal range of $2-10 \mathrm{~nm}$ is used as a reference. These samples are calibrated concerning the mass deposition of oxygen by means of a reference-free X-ray fluorescence analysis at the plane-grating monochromator beamline for undulator radiation; this calibration takes place at the PTB laboratory at the BESSY II synchrotron facility [13-16]. The oxygen mass depositions of all samples are listed in Table 1. The first step in each quantification procedure is to measure the set of reference samples in the XRF/XPS instrument by means of XRF. Detector response function, background contributions and
Table 1. Calibration results of the reference samples determined by reference free X-ray fluorescence analysis.

\begin{tabular}{ll}
\hline Sample & $\begin{array}{l}\text { Mass deposition oxygen } \\
{\left[\mathrm{O} / \mathrm{ngcm}^{-2}\right]}\end{array}$ \\
\hline Reference sample 1 & $439(36)$ \\
Reference sample 2 & $682(56)$ \\
Reference sample 3 & $868(71)$ \\
Reference sample 4 & $1100(90)$ \\
Reference sample 5 & $1192(97)$ \\
\hline
\end{tabular}

fluorescence lines are convoluted and then fitted to the obtained spectra from each sample [17]. The contributions to the spectrum are fitted and the count rates of the oxygen $\mathrm{O} \mathrm{K} \alpha$ line and silicon resonant Raman scattering (RRS $(\mathrm{Si})$ ) is determined [14]. The resonant Raman scattering of silicon originates mainly from the bulk and is used as a monitoring signal; this is necessary due to the insufficient stability of the X-ray source. The ratios of the O K $\alpha$ to RRS (Si) of each sample and the mass deposition of oxygen from the calibration are then used to fit a curve for the determination of the mass deposition of oxygen on the sphere. In [10] a logarithmic function originating from the fundamental parameter approach was fitted as the calibration curve. Here, as the oxide layers in this study are very thin, linear behavior can be assumed and linear regression is applied to the calibration points. In the calibration function (1) $d_{\mathrm{O}}$ is the mass deposition of oxygen, $P_{1}$ is the calibration factor, and $R_{\mathrm{O} / \mathrm{RRS}}$ is the ratio of the $\mathrm{O} K \alpha$ count rate to the $\mathrm{RRS}(\mathrm{Si})$ count rate.

$$
d_{\mathrm{O}}=P_{1} \cdot R_{\mathrm{O} / \mathrm{RRS}}
$$

\subsubsection{XRF measurement of the silicon sphere}

After the calibration curve is established, the actual measurement of the sphere via XRF takes place. The spectra from each measured point are fitted as well to determine the ratio of $\mathrm{O} \mathrm{K} \alpha$ to $\mathrm{RRS}(\mathrm{Si})$, which is then used in equation (1) to obtain the mass deposition of oxygen $d_{\mathrm{O}}$. This results in the mass deposition of oxygen at every point measured on the sphere. However, as the surface layer consists not only of silicon oxide but also of a water layer and a carbonaceous contamination layer, XRF measurements alone are not sufficient for quantification. Due to the presence of oxygen in all sublayers, it is necessary to attribute the right amount of oxygen to each sublayer. This is achieved via the following XPS measurements.

\subsubsection{XPS measurement of the sphere}

The concentration of oxygen, carbon and oxidized silicon in the surface layer is determined via XPS. As solely the ratios of element concentrations to each other are investigated here (i.e. no absolute masses or depositions), no reference samples are used. Photoemission lines of the elements present ( $\mathrm{Si} 2 p, \mathrm{O} 1 s, \mathrm{C} 1 s)$ are scanned and fitted via 
a Gauss-Lorentz fit to determine each peak area [18]. Furthermore, the peak areas are normalized by means of the photoelectric cross sections [19], the inelastic mean free path of the photoelectrons [20] and the transmission function of the spectrometer [21]. The atomic fraction of each element is then calculated from the normalized peak areas [10].

The binding energy of oxidized silicon is shifted towards higher values, thus allowing silicon bound in oxide to be distinguished from the silicon in the bulk. Only oxidized silicon is considered a part of the surface layer. The $\mathrm{C} 1 \mathrm{~s}$ peak shows three different contributions that can be attributed to certain chemical binding states. This allowed the amount of oxygen and hydrogen bound in the carbonaceous layer to be estimated, which is explained in detail in [10].

\subsubsection{Determination of surface masses by combination of XRF and XPS}

In the last step, the mass deposition determined via $\mathrm{XRF}$ and the elemental ratios are combined to determine the mass contributions of each sublayer and the overall surface layer. The mass deposition of oxygen $d_{\mathrm{O}}$ combined with the ratio of the XPS elemental atomic fractions of $\mathrm{SiO}_{2}$ and the remaining oxygen yields the mass deposition of silicon and oxygen in the whole surface. The amount of carbon is derived from the ratio of carbon to oxygen and $d_{\mathrm{O}}$. As hydrogen is not detectable via XPS, a worst-case approximation for the carbonaceous layer is made, as well as a correction for silicon hydroxide at the interface of the oxide layer and the water layer. The calculation of sublayers requires additional assumptions and is derived from the overall surface layer. Thus, the relative measurement uncertainty of the sublayers is higher than the uncertainty of the overall surface layer [10].

\subsubsection{Number of measuring points}

We want to achieve a good representation of the surface layer without the need to cover the entire surface are of the sphere with measurement spots. To determine the sufficient number of measuring points on a sphere, earlier measurements on three different ${ }^{28} \mathrm{Si}$-spheres are used to estimate a preliminary value for the standard deviation of the carbonaceous layer. The carbonaceous contamination layer is chosen because it is the layer with the largest variation and because it is expected that the cleaning will have the strongest influence on this sublayer. The maximal observed relative standard deviation is $13 \%$. The sample size formula for estimation a population mean is $n=(Z \cdot \sigma / \Delta)^{2}$. For a confidence level of $95 \% Z$ equals 1.96. To ensure a precision $\Delta$ of $5 \%$ twenty-six points are necessary. However, 42 points were chosen because of the potential better distribution on the surface.

As described in [10], the sphere manipulator of the $\mathrm{XRF} / \mathrm{XPS}$ instrument has two rotational axes available to reach every point on the sphere. One motorized axis in the center moves the sphere around its vertical axis. The sphere lies in the center of the manipulator on three PEEK balls, allowing the sphere to be rolled around an axis in the horizontal plane. The PEEK axis realizes the movement of the sphere by turning one of the pulleys, and thus via a movement relative to the sphere. When choosing a distribution pattern for the measurement points, the movement of the PEEK axis should be reduced to a minimum in order to reduce the risk of changing the surface of the sphere. The measuring points are arranged along eight great circles in such a way that the angular distance of the points in the pole region is bigger than in the equator region. Hence, each measurement point represents a patch of the same surface area. The large circles are realized via the PEEK axis, while the more frequent movements are performed via the vertical axis.

\section{Results and discussion}

\subsection{Mass measurements}

Before each measurement, the test spheres are cleaned according to the cleaning procedure described above (see Sect. 2.1). Each sphere is cleaned and measured between six and eight times (42 cleanings and mass measurements in total). After applying the cleaning procedure, the mass of silicon spheres changes according to a non-linear function of time [9]. The mass results corresponding to the last measurement before the surface quantification were chosen for the evaluation of the repeatability in order to ensure a good comparability between the two independent methods. For each test sphere and each measurement, the mass difference to the mean value of the measured mass with respect to the reference sphere $(\mathrm{Sm} 14)$ is determined; these differences are listed in Table 2. A diagram of the results is shown in Figure 2. Before the first series, between the first and the second series and after the second series, the mass of the reference spheres Sm14 and Si12-06 in vacuum is determined in such a way that it could be traced to the national prototype no. 52 in air. Mass stability within \pm 0.7 and $\pm 0.9 \mu \mathrm{g}$ was observed for the Sm14 and Si12-06 spheres, respectively. The mass stability of the Sm14 reference sphere in the course of the measurements in vacuum in comparison to the Si12-06 reference sphere is determined to be within $\pm 0.3 \mu \mathrm{g}$ for both series 1 and 2 .

The curves in Figure 2 show the mass variation of the spheres after the different cleanings. The observed difference between the minimum and the maximum mass value of a single sphere ranges between 1.2 and $4.6 \mu \mathrm{g}$. The maximum deviation from the mean value observed for all spheres amounts to $2.9 \mu \mathrm{g}$. From one cleaning to the next, the average mass change of all spheres was determined to be $-0.45 \mu \mathrm{g}$ with a standard deviation of $0.44 \mu \mathrm{g}$. The difference between the mean values of the series 1 and series 2 amounts to $1.4 \mu \mathrm{g}$, which may be caused by a change of the mass of the reference sphere due to the second cleaning. Although the results of the mass determination of the Sm14 before the first and between the first and the second series indicate a possible instability of about $1 \mu \mathrm{g}$, this is not considered to be sufficient as a proof. Therefore, a correction based on the mass difference between mean values of the series was not applied. In order to give a measure for the mass variations after the cleaning, 


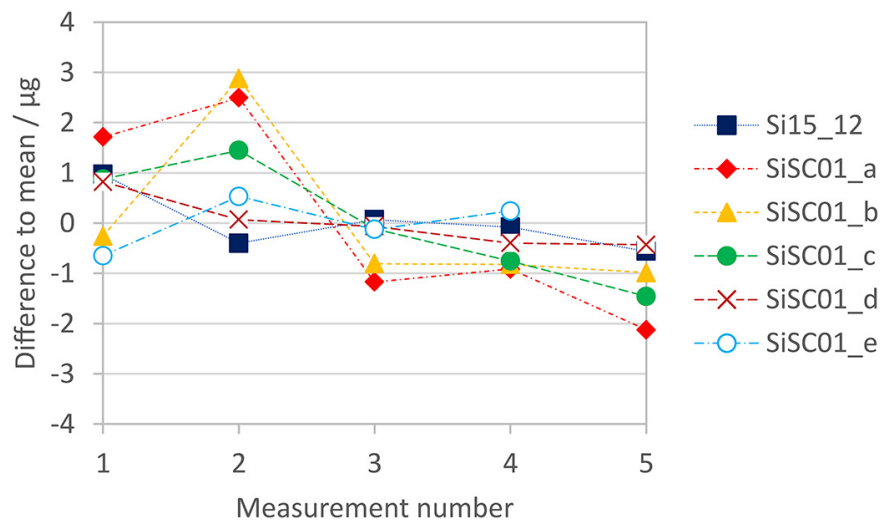

Fig. 2. Diagram of the data given in table 3.2.1 for the measured mass differences between the test spheres and the reference sphere (Sm14). The individual mass differences of a sphere are given with respect to their observed mean value $(\bar{m})$ [9].

the standard deviation of the results for the different test spheres and the pooled standard deviation of all results are given in Table 3 . The results reveal that the repeatability of the applied cleaning procedure is in the order of $2 \mu \mathrm{g}$ [9]. This value is in the order of magnitude of the standard uncertainty $(k=1)$ of the measured mass differences given in Table 2. It is estimated to be about one microgram and considers the influence of the repeatability of loading and unloading, but not the influence of the cleaning process.

\subsection{Surface measurements}

\subsubsection{Repeatability of the quantification method}

As the cleaning procedure is investigated in terms of repeatability, knowledge of the repeatability of the surface quantification method itself is required. Therefore, a sphere is measured consecutively eleven times. Between the measurements the sphere is extracted to the load lock chamber, which is vented with nitrogen, opened for max. $30 \mathrm{~s}$, then closed and immediately evacuated. Thus, between the measurements the sphere is exposed to a change from vacuum to ambient conditions of the laboratory. After evacuating the load lock the sphere is again inserted to the analysis chamber. The results are listed in Table 4 and shown in Figure 3. The standard deviation of the surface layer mass is $1.4 \mu \mathrm{g}$ (1.7\%) The systematic mass gain of approximately $3 \mu \mathrm{g}$ within the first measurements is included in the calculation of the standard deviation as the gain is considered inherent to the usual handling of the measurement procedure. The relative standard deviations of the sublayers are $6.8 \%$ for the carbonaceous contamination layer and $10.7 \%$ for the water layer due to the sublayers being derived from the overall surface layer. A mass growth of the carbonaceous layer and thus, the surface layer is observable during the first measurement and evacuation cycles. The mass stabilizes after measurement six. In contrast, Table 5 shows the repeatability of the interim values (e.g. the mass deposition of oxygen $d_{\mathrm{O}}$ and the atomic concentrations of silicon, carbon and oxygen). These values are derived from the XRF or XPS spectra and peak fit and have not been 
Table 3. Standard deviation of the observed mass variations after cleaning given for the individual test spheres and as pooled standard deviation for all gravimetric measurements.

\begin{tabular}{lllllll}
\hline \multicolumn{9}{c}{ Standard deviation $(\mu \mathrm{g})$} & & Pooled standard deviation $(\mu \mathrm{\mu g})$ \\
\hline Si15_12 & SiSC01_a & SiSC01_b & SiSC01_c & SiSC01_d & SiSC01_e & \\
\hline 0.6 & 2.0 & 1.6 & 1.2 & 0.5 & 0.5 & 1.2 \\
\hline
\end{tabular}

Table 4. Repeatability of the surface quantification method investigated with eleven measurements on one sphere.

\begin{tabular}{lllc}
\hline & Mean $(\mu \mathrm{g})$ & Standard deviation $(\mu \mathrm{g})$ & Relative standard deviation $(\%)$ \\
\hline SL & 79.4 & 1.4 & 1.7 \\
OL & 55.8 & 1.7 & 3.0 \\
CL & 13.6 & 0.9 & 6.8 \\
WL & 10.1 & 1.7 & 10.7 \\
\hline
\end{tabular}
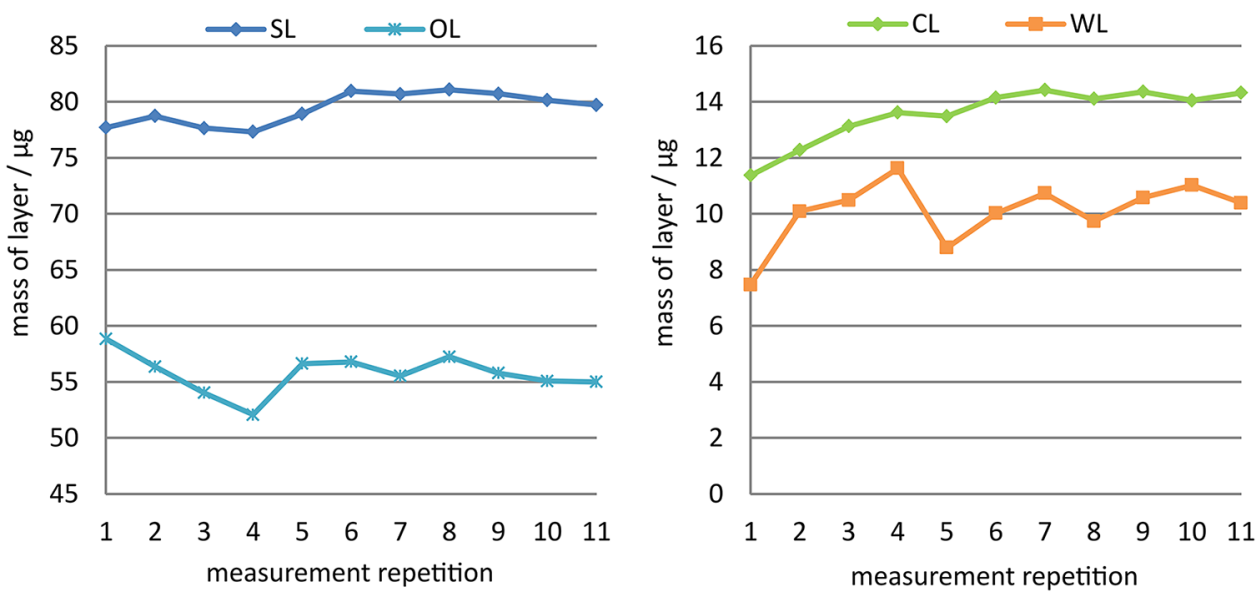

Fig. 3. Results of the repeated measurements for surface layer, oxide layer, carbonaceous layer and water layer. The carbonaceous layer shows growth within the first three cycles of transfer, venting, evacuating and measurement.

Table 5. Repeatability of the interim values before the combination of XRF and XPS data.

\begin{tabular}{llll}
\hline & Mean & Standard deviation & Relative standard deviation (\%) \\
\hline $\mathrm{d}_{\mathrm{O}}$ & $139.5 \mathrm{ng} / \mathrm{cm}^{2}$ & $2.3 \mathrm{ng} / \mathrm{cm}^{2}$ & 1.7 \\
$\mathrm{c}_{\mathrm{SiO} 2}$ & $19.9 \mathrm{at} \%$ & $0.3 \mathrm{at} \%$ & 1.3 \\
$\mathrm{c}_{\mathrm{SiOx}}$ & $20.9 \mathrm{at} \%$ & $0.8 \mathrm{at} \%$ & 4.0 \\
$\mathrm{c}_{\mathrm{C}}$ & $19.6 \mathrm{at} \%$ & $1.1 \mathrm{at} \%$ & 5.6 \\
$\mathrm{c}_{\mathrm{O}}$ & $59.5 \mathrm{at} \%$ & $0.6 \mathrm{at} \%$ & 0.9 \\
\hline
\end{tabular}

combined yet, thus allowing the repeatability of the input data before the actual calculation to be evaluated. The relative standard deviation is $0.9 \%$ for the atomic concentration of oxygen and $5.6 \%$ for the concentration of carbon. The repeatability of the mass deposition of oxygen shows a standard deviation of $1.7 \%$.

\subsubsection{Surface layers after cleaning}

As mentioned above, the measurements used to investigate the cleaning procedure are carried out in two series. The calibration factor $P_{1}$ is $1.78 \times 10^{-6}$ for the first series and $1.76 \times 10^{-6}$ for the second, which is an agreement within $1 \%$.

All results of the sphere measurements are shown in Table 6 . The diagram in Figure 4 shows the results of the surface, oxide and carbon layer values for all measurements. The calculation of sublayers requires additional assumptions and is derived from the overall surface layer. Thus, the relative measurement uncertainty of the sublayers is higher than the uncertainty of the overall surface layer. While the surface layer SL shows a relative standard uncertainty of $11 \%$, it is $13 \%$ for the oxide layer 
Table 6. Results of the surface quantification with combined XRF and XPS.

\begin{tabular}{|c|c|c|c|c|c|c|}
\hline \multirow{5}{*}{ Si15_12 } & Date & 04.05 .2017 & 15.05 .2017 & 11.09 .2017 & 19.09.2017 & 09.10 .2017 \\
\hline & $\mathrm{OL}(\mu \mathrm{g})$ & $48.8(7.1)$ & $51.9(7.2)$ & $47.3(6.9)$ & $54.0(7.5)$ & $53.8(7.5)$ \\
\hline & CL $(\mu \mathrm{g})$ & $18.9(4.3)$ & $17.8(4.2)$ & $16.9(4.2)$ & $19.0(4.5)$ & $19.1(4.4)$ \\
\hline & WL $(\mu \mathrm{g})$ & $10.0(3.2)$ & $7.8(3.1)$ & $11.4(3.2)$ & $7.7(3.3)$ & $7.0(3.3)$ \\
\hline & $\mathrm{SL}(\mu \mathrm{g})$ & $77.0(8.3)$ & $76.5(8.3)$ & $75.3(8.0)$ & $79.8(8.5)$ & $78.7(8.5)$ \\
\hline \multirow{5}{*}{ SiSC01_a } & Date & 08.05 .2017 & 15.05 .2017 & 12.09 .2017 & 20.09 .2017 & 10.10 .2017 \\
\hline & $\mathrm{OL}(\mu \mathrm{g})$ & $63.0(8.4)$ & $64.8(8.8)$ & $66.8(8.6)$ & $62.6(8.2)$ & $62.5(8.3)$ \\
\hline & $\mathrm{CL}(\mu \mathrm{g})$ & $18.2(4.8)$ & $20.3(5.1)$ & $16.8(4.8)$ & $16.7(4.7)$ & $17.7(4.8)$ \\
\hline & $\mathrm{WL}(\mu \mathrm{g})$ & $8.5(3.6)$ & $8.2(3.8)$ & $7.5(3.7)$ & $8.8(3.6)$ & $8.0(3.6)$ \\
\hline & $\mathrm{SL}(\mu \mathrm{g})$ & $87.9(9.4)$ & $93(10)$ & $90.1(9.4)$ & $88.1(9.2)$ & $88.3(9.3)$ \\
\hline \multirow{5}{*}{ SiSC01_b } & Date & 08.05 .2017 & 16.05 .2017 & 13.09 .2017 & 21.09 .2017 & 11.10 .2017 \\
\hline & $\mathrm{OL}(\mu \mathrm{g})$ & $59.8(8.1)$ & $67.3(8.7)$ & $62.1(8.2)$ & $67.8(8.7)$ & $64.6(8.4)$ \\
\hline & $\mathrm{CL}(\mu \mathrm{g})$ & $17.8(4.7)$ & $18.7(4.9)$ & $16.9(4.7)$ & $16.8(4.8)$ & $16.7(5.0)$ \\
\hline & $\mathrm{WL}(\mu \mathrm{g})$ & $8.6(3.6)$ & $5.2(3.7)$ & $8.7(3.6)$ & $5.0(3.6)$ & $7.1(3.6)$ \\
\hline & $\mathrm{SL}(\mu \mathrm{g})$ & $86.2(9.3)$ & $91.2(9.6)$ & $87.7(9.3)$ & $89.5(9.5)$ & $87.7(9.5)$ \\
\hline \multirow{5}{*}{ SiSC01_c } & Date & 09.05 .2017 & 18.05 .2017 & 14.09 .2017 & 22.09 .2017 & 12.10 .2017 \\
\hline & $\mathrm{OL}(\mu \mathrm{g})$ & $51.4(7.5)$ & $61.0(8.1)$ & $66.9(8.6)$ & $60.7(8.1)$ & $62.0(8.2)$ \\
\hline & $\mathrm{CL}(\mu \mathrm{g})$ & $18.1(4.9)$ & $16.9(4.7)$ & $16.1(4.7)$ & $16.7(4.6)$ & $16.8(4.6)$ \\
\hline & $\mathrm{WL}(\mu \mathrm{g})$ & $14.4(3.6)$ & $9.3(3.6)$ & $7.1(3.6)$ & $8.6(3.5)$ & $7.9(3.5)$ \\
\hline & $\mathrm{SL}(\mu \mathrm{g})$ & $83.8(9.1)$ & $87.2(9.2)$ & $89.1(9.4)$ & $85.9(9.2)$ & $86.6(9.2)$ \\
\hline \multirow{5}{*}{ SiSC01_d } & Date & 10.05 .2017 & 19.05 .2017 & 15.09 .2017 & 05.10 .2017 & 16.10 .2017 \\
\hline & $\mathrm{OL}(\mu \mathrm{g})$ & $61.7(8.2)$ & $60.2(8.1)$ & $64.2(8.4)$ & $68.2(8.6)$ & $65.6(8.5)$ \\
\hline & $\mathrm{CL}(\mu \mathrm{g})$ & $16.3(4.6)$ & $16.4(4.5)$ & $15.7(4.6)$ & $15.1(4.7)$ & $15.2(4.5)$ \\
\hline & $\mathrm{WL}(\mu \mathrm{g})$ & $8.2(3.5)$ & $8.5(3.5)$ & $7.4(3.5)$ & $7.2(3.6)$ & $6.3(3.5)$ \\
\hline & $\mathrm{SL}(\mu \mathrm{g})$ & $86.1(9.2)$ & $85.1(9.1)$ & $86.9(9.3)$ & $89.4(9.3)$ & $85.8(9.3)$ \\
\hline \multirow{5}{*}{ SiSC01_e } & Date & 11.05 .2017 & 22.05 .2017 & 18.09 .2017 & 06.10 .2017 & \\
\hline & $\mathrm{OL}(\mu \mathrm{g})$ & $56.7(8.1)$ & $68.0(8.9)$ & $64.2(8.8)$ & $65.7(8.9)$ & \\
\hline & $\mathrm{CL}(\mu \mathrm{g})$ & $21.6(5.0)$ & $20.6(5.1)$ & $19.3(5.0)$ & $20.2(5.1)$ & \\
\hline & $\mathrm{WL}(\mu \mathrm{g})$ & $11.1(3.8)$ & $7.1(3.9)$ & $8.3(3.8)$ & $8.4(3.9)$ & \\
\hline & $\mathrm{SL}(\mu \mathrm{g})$ & $89.3(9.4)$ & $94.3(9.8)$ & $92(10)$ & $94(10)$ & \\
\hline
\end{tabular}

Table 7. Standard deviation of the surface layer mass changes for each sphere and pooled standard deviation of all measurements.

\begin{tabular}{lllllll}
\hline \multicolumn{9}{c}{ Standard deviation $(\mu \mathrm{g})$} & Pooled standard deviation $(\mu \mathrm{g})$ \\
\cline { 1 - 5 } Si15_12 & SiSC01_a & SiSC01_b & SiSC01_c & SiSC01_d & SiSC01_e & \\
\hline 1.8 & 2.1 & 1.9 & 1.9 & 1.6 & 2.3 & 2.0 \\
\hline
\end{tabular}

OL, $25 \%$ for the carbonaceous layer CL and $47 \%$ for the water layer WL. Figure 5 shows an example of the surface layer masses with standard uncertainties for one sphere (SiSC01 a). The oxygen mass deposition of this sphere is also shown in the diagram. As expected, the values for the SL are mainly influenced by the mass deposition of oxygen. The average correlation factor for all spheres is 0.6 , while it is greater than 0.8 for three of the spheres (SiSC01_a, $-b$, and $-\mathrm{d})$. The observed difference between the minimum and the maximum deviation from the mean SL value of a single sphere ranges between 3.1 and $5.1 \mu \mathrm{g}$. The maximum deviation from the mean value observed for all spheres amounts to $3.3 \mu \mathrm{g}$.

The standard deviation of the results of the different test spheres and the pooled standard deviation of all results provide a measure of the variations of the surface layer mass and are listed in Table 7 . The pooled standard deviation considers the different numbers of measurement 

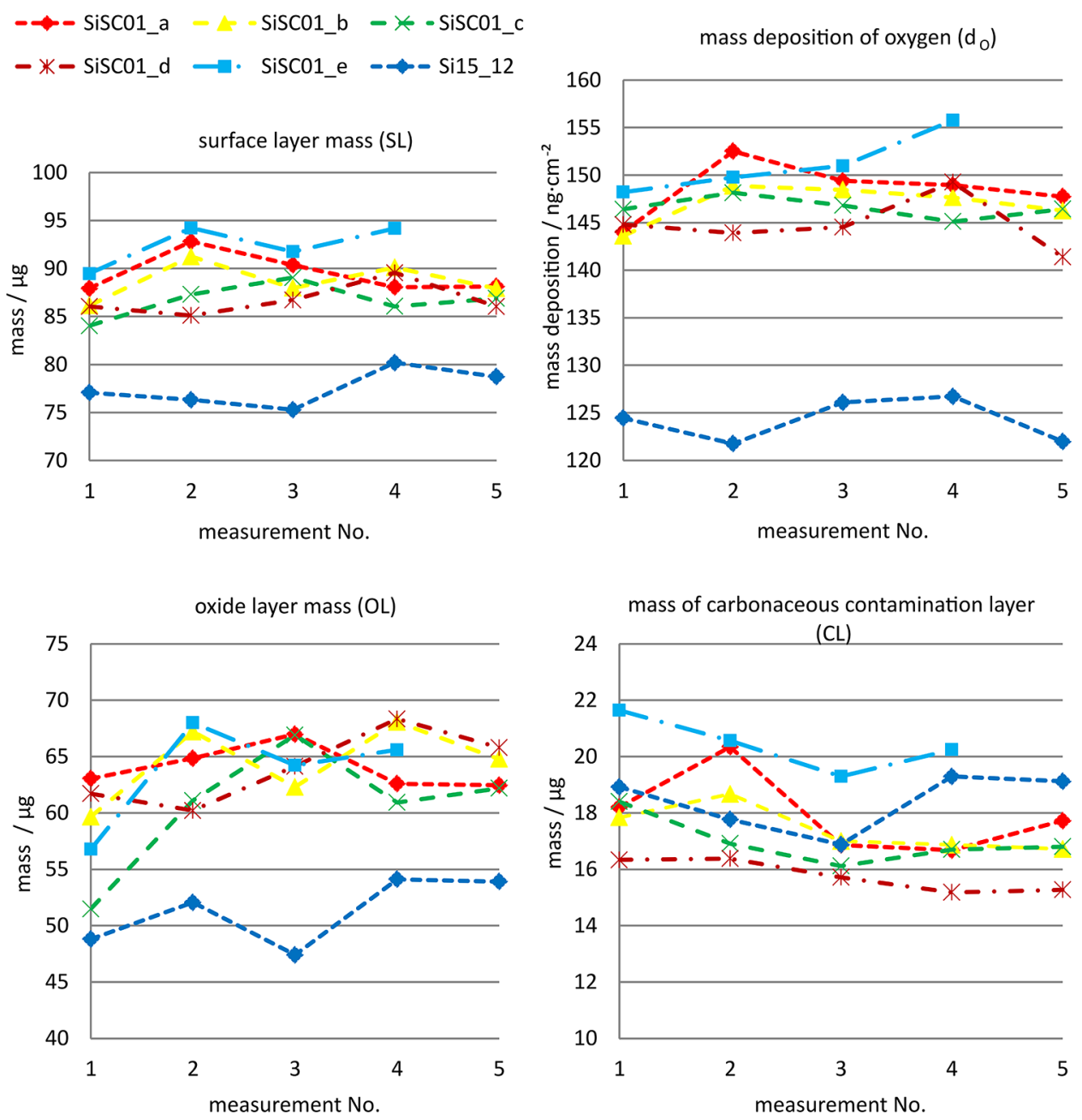

Fig. 4. Results for SL, OL, CL and the oxygen mass deposition for all spheres and all measurements.

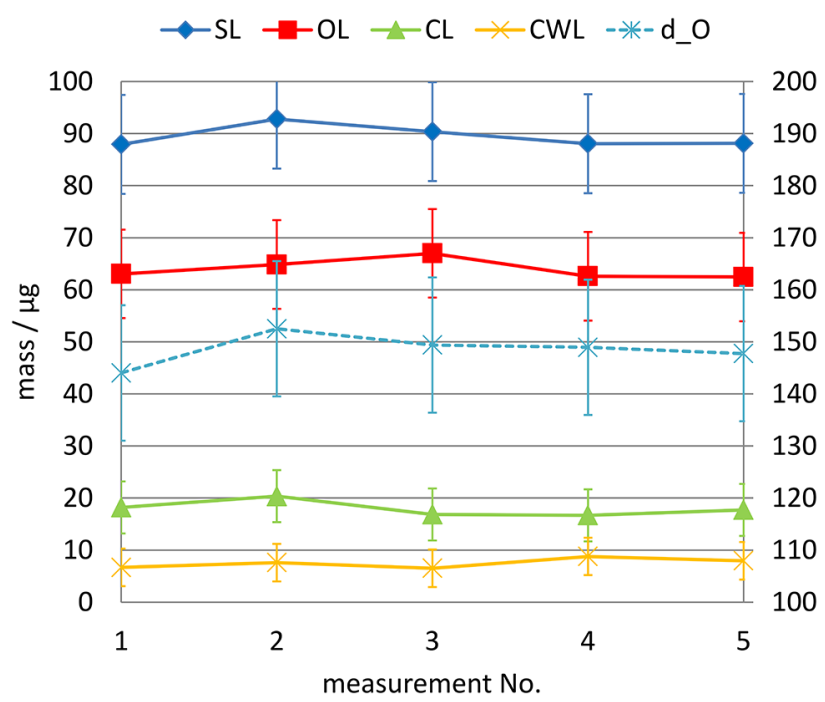

Fig. 5. Results with standard uncertainty for the sphere SiSC01_a with the surface layer (SL), oxide layer (OL), carbonaceous contamination layer $(\mathrm{CL})$, water layer $(\mathrm{WL})$ and the oxygen mass deposition. of the spheres. The surface layer standard deviation for all measurements is $2.0 \mu \mathrm{g}$ and can be used to assess the repeatability of the cleaning procedure.

\subsection{Repeatability}

The results of the mass measurement reveal that the repeatability of the applied cleaning procedure is better than $2 \mu \mathrm{g}$, which is in the order of magnitude of the measurement uncertainty. The repeatability of the surface layer mass after cleaning acquired by XRF and XPS measurements is $2.0 \mu \mathrm{g}$ and only slightly worse than the repeatability of the measurement technique, which is $1.4 \mu \mathrm{g}$. With a correlation coefficient of 0.3 for all corresponding mass and surface measurements there is a weak correlation between the change of the surface layer mass and the corresponding mass values of the spheres. Considering the repeatability and measurement uncertainty of the quantification method, the cleaning of the sphere had only a negligible influence on the results for the surface layer mass of the spheres. 


\section{Summary and conclusion}

The cleaning procedure for silicon spheres applied at PTB was investigated by means of gravimetric measurements in vacuum and a spectroscopic quantification of the surface layer based on XRF and XPS. A vacuum transfer system between the mass comparator and the surface measurement instrument prevented the surface layer (and therefore the mass) from changing due to exposure to ambient conditions.

A repeatability in the order of magnitude of the measurement uncertainty for mass measurements and the repeatability of the surface quantification method lead to the conclusion that the cleaning method is state of the art and needs currently no improvement to meet the targeted uncertainties. It can be summed up that this uncertainty contribution is not significant in comparison to the present uncertainty of the realization of the new definition of the kilogram by means of silicon spheres.

The authors would like to thank Dr. Frank Härtig and Dr. Horst Bettin for their support in this research project, Dr. Matthias Müller for his valuable discussions on this topic and all our colleagues involved in manufacturing the spheres.

\section{References}

1. Bureau International des Poids et Mesures: Resolutions of the 26th meeting of the General Conference of Weights and Measures (CGPM), Versailles, 13-16 November 2018, https://www.bipm.org/utils/common/pdf/CGPM-2018/ 26th-CGPM-Resolutions.pdf

2. Bureau International des Poids et Mesures: Mise en pratique for the definition of the kilogram in the new SI, 2019. SI Brochure 9th edition Appendix 2, https://www.bipm.org/ utils/en/pdf/si-mep/SI-App2-kilogram.pdf

3. Bureau International des Poids et Mesures: The International System of Units (SI) (2019). 9th SI Brochure: https:// www.bipm.org/utils/common/pdf/si-brochure/SI-Brochure9-EN.pdf

4. Y. Azuma et al., Improved measurement results for the Avogadro constant using a 28Si-enriched crystal, Metrologia 52, 360-375 (2015)

5. M.P. Seah, S.J. Spencer, Ultrathin $\mathrm{SiO} 2$ on Si. I. Quantifying and removing carbonaceous contamination, J. Vac. Sci. Technol. A 21, 345-352 (2003)

6. H. Bettin, D. Schiel, M. Vogtmann, H. Niemann, Cleaning of silicon density standards, in: Proceedings XIX IMEKO World Congress 1, 179-181 (2009)
7. A. Picard, P. Barat, M. Borys, M. Firlus, S. Mizushima, State-of-the-art mass determination of $28 \mathrm{Si}$ spheres for the Avogadro project, Metrologia 48, 112-119 (2011)

8. J.W. Chung, V. Görlitz, M. Vogtmann, E. Beyer, F. Härtig, The PTB Cleaning Procedure for Silicon Spheres. Physikalisch-Technische Bundesanstalt, Braunschweig, 2016

9. M. Mecke, M. Borys, E. Beyer, Investigation of the repeatability of a cleaning method for silicon spheres by gravimetric measurements, ACTA IMEKO (accepted for publication), (2020)

10. M. Müller, B. Beckhoff, E. Beyer, E. Darlatt, R. Fliegauf, G. Ulm, M. Kolbe, Quantitative surface characterization of silicon spheres by combined XRF and XPS analysis for the determination of the Avogadro constant, Metrologia 54, 653-662 (2017)

11. G. Bartl et al. Counting the atoms in a new $28 \mathrm{Si}$ single crystal for the new kilogram definition, Metrologia 54, 693-715 (2017)

12. M. Kolbe, B. Beckhoff, M. Krumrey, G. Ulm, Thickness determination for $\mathrm{Cu}$ and $\mathrm{Ni}$ nanolayers: comparison of reference-free fundamental-parameter based x-ray fluorescence analysis and x-ray reflectometry, Spectrochim. Acta B 60, 505-510 (2005)

13. P. Hönicke, M. Kolbe, M. Krumrey, R. Unterumsberger, B. Beckhoff, Experimental determination of the oxygen K-shell fluorescence yield using thin $\mathrm{SiO}_{2}$ and $\mathrm{Al}_{2} \mathrm{O}_{3}$ foils, Spectrochim. Acta B 124, 94-98 (2016)

14. M. Müller, B. Beckhoff, G. Ulm, B. Kanngießer, Absolute determination of cross sections for resonant Raman scattering on silicon, Phys. Rev. A 7, 3147-3159 (2006)

15. B. Beckhoff, Reference-free x-ray spectrometry based on metrology using synchrotron radiation, J. Anal. Atom. Spectrom. 23, 845-853 (2008)

16. M. Müller, P. Hönicke, B. Detlefs, C. Fleischmann, Characterization of high-k nanolayers by grazing incidence x-ray spectrometry, Materials 7, 3147-3159 (2014)

17. F. Scholze, M. Procop, Modelling the response function of energy dispersive $\mathrm{x}$-ray spectrometers with silicon detectors, X-Ray Spectrom. 38, 312-321 (2009)

18. R. Hesse, Unifit 2017, Unifit Scientific SoftwareGmbH, Leipzig, 2017. http://unifit-software.de/

19. J.H. Scofield, Hartree-Slater subshell photoionizationcrosssections at1254 and $1487 \mathrm{eV}$, J. Electron Spectros. Relat. Phenomena 8, 129-137 (1976)

20. S. Tanuma, C.J. Powell, D.R. Penn, Calculations of electron inelastic mean free paths (IMFPS). IV. Evaluation of calculated IMFPs and of the predictive IMFP formula TPP-2 for electron energies between 50 and2000 eV, Surf. Interface Anal. 20, 77-89 (1993)

21. R. Hesse, P. Streubel, R. Szargan, Improved accuracy of quantitative XPS analysis using predetermined spectrometer transmission functions with UNIFIT 2004, Surf. Interface Anal. 37, 589-607 (2005)

Cite this article as: Edyta Beyer, Michael Borys, Michael Mecke, Michael Kolbe, Investigation of a cleaning procedure for silicon spheres used in the realization and dissemination of the redefined kilogram via combined spectroscopic and gravimetric measurements, Int. J. Metrol. Qual. Eng. 11, 18 (2020) 